\title{
Type 2 Diabetes, Metabolic Syndrome, Lipid Metabolism
}

\author{
Jacques Beltrand and Claire Levy-Marchal \\ INSERM, Unité 690, Hôpital Robert-Debré, et Université Paris Diderot, Paris, France
}

\section{New paradigm: brain regulation of glucose and energy homeostasis}

\section{AMPK is essential for energy homeostasis regulation and glucose sensing by POMC and AgRP neurons}

Claret M, Smith MA, Batterham RL, Selman C, Choudhury Al, Fryer LG, Clements M, Al-Qassab H, Heffron H, Xu AW, Speakman JR, Barsh GS, Viollet B, Vaulont S, Ashford ML, Carling D, Withers DJ

Centre for Diabetes and Endocrinology, Rayne Institute, University College London, London, UK

J Clin Invest 2007;117:2325-23436

Background: Hypothalamic AMP-activated protein kinase (AMPK) has been suggested to act as a key sensing mechanism, responding to hormones and nutrients in the regulation of energy homeostasis. However, the precise neuronal populations and cellular mechanisms involved are unclear. The effects of long-term manipulation of hypothalamic AMPK on energy balance are also unknown.

Methods: To directly address such issues, the authors generated POMC $-\alpha_{2} / \mathrm{KO}$ and POMC- $\alpha_{2} / \mathrm{KO}$ mice lacking AMPK- $\alpha_{2}$ in proopiomelanocortin (POMC)- and agouti-related protein (AgRP)-expressing neurons, key regulators of energy homeostasis.

Results: POMC- $\alpha_{2} / \mathrm{KO}$ mice developed obesity due to reduced energy expenditure and dysregulated food intake but remained sensitive to leptin. In contrast, POMC- $\alpha_{2} / \mathrm{KO}$ mice developed an age-dependent lean phenotype with increased sensitivity to a melanocortin agonist. Electrophysiological studies in AMPK- $\alpha_{2}$-deficient POMC or AgRP neurons revealed normal leptin or insulin action but absent responses to alterations in extracellular glucose levels, showing that glucose-sensing signaling mechanisms in these neurons are distinct from those pathways utilized by leptin or insulin.

Conclusion(s): Taken together with the divergent phenotypes of POMC $-\alpha_{2} / \mathrm{KO}$ and AgRP- $\alpha_{2} / \mathrm{KO}$ mice, the data suggest that while AMPK plays a key role in hypothalamic function, it does not act as a general sensor and integrator of energy homeostasis in the mediobasal hypothalamus.

Using genetically modified mice, this paper demonstrates that AMP-kinase is not a general sensor for the regulation of energy homeostasis in the hypothalamus as it mediates glucose-sensing signaling mechanisms but is not involved in the leptin and insulin pathways. AMPK is an enzyme involved in pathways that generate ATP, and participates in energy homeostasis in response to nutrients and hormonal signals. In peripheral tissues, it is involved in catabolic pathways (fat oxidation, lipolysis and suppression of hepatic glucose production). In the central nervous system, AMPK is seen as a general integrator of energy homeostasis. Anorexigenic signals such as leptin, insulin and glucose inhibit AMP activity, promoting food intake (fig. 1).

The different neuronal populations were not identified in the hypothalamus. The generation of two specific-KO mouse models (deficient in AMPK activity in POMC and AgRP neurons) shows two different phenotypes, indicating a clear dissociation between the signaling mechanisms by which POMC and AgRP neurons sense glucose and hormonal signals: the effects of leptin and insulin were not affected by AMPK activity suppression. Electrophysiological studies confirm that APMK is not necessary for the effects of leptin and insulin. Conversely, glucose sensing is abolished in both neuron populations when AMPK activity is suppressed and the mechanism for this remains unknown but does not appear to be only a general protective one. Glucose levels in the hypothalamus are involved in the orexigenic process via GLUT2, K-ATP channels and glucokinase [2-4] with complex relations between electrophysiological activity and physiological response.

For more on the subject, read the chapter on 'Neuroendocrinology' prepared by Nicolas de Roux. 


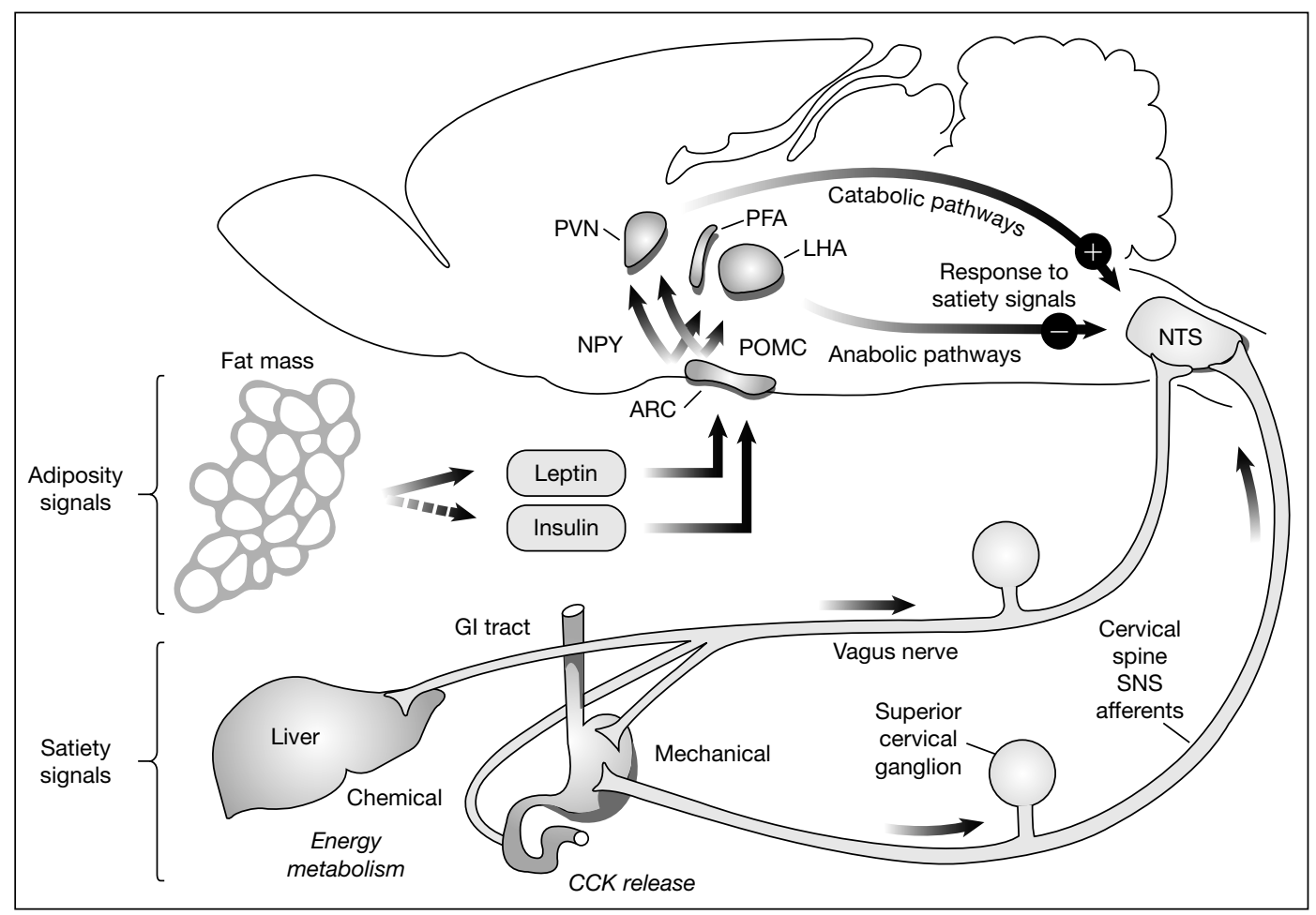

Fig. 1. CNS and regulation of food intake and energy homeostasis. From Schwartz et al. [1].

\section{Brain IRS2 signaling coordinates lifespan and nutrient homeostasis}

Taguchi A, Wartschow LM, White MF

Howard Hughes Medical Institute, Division of Endocrinology, Children's Hospital Boston, Harvard Medical School,

Boston, Mass., USA

Science 2007:317:369-372

Background: Reduced insulin-like signaling extends the lifespan of Caenorhabditis elegans and Drosophila. Methods: Alteration in the insulin-signaling pathway was generated using general or brain-specific invalidation of $\operatorname{Irs} 2$.

Results: In mice, less insulin receptor substrate-2 (Irs2) signaling throughout the body or just in the brain extended lifespan up to $18 \%$. At 22 months of age, brain-specific Irs 2 knockout mice were overweight, hyperinsulinemic, and glucose intolerant; however, compared with control mice, they were more active and displayed greater glucose oxidation, and during meals they displayed stable superoxide dismutase2 concentrations in the hypothalamus.

Conclusion(s): Less Irs2 signaling in aging brains can promote healthy metabolism, attenuate meal-induced oxidative stress, and extend the lifespan of overweight and insulin-resistant mice.

Caloric restriction increases lifespan and insulin sensitivity in animals whereas reduced insulin signaling extends lifespan in C. elegans and Drosophila. Modulation of insulin signaling is used here by generating a mouse model with partial or total invalidation of Irs 2 in all tissues (Irs $2+/-$ ) or specific to the brain (b/rs2-I- and b/rs2+/-). Adult Irs2+/- mice are more insulin-sensitive and more glucosetolerant with extended live span. Both brain models are insulin-resistant and glucose-intolerant together with a significant reduction in lifespan. These changes were associated with improved nutrient utilization and less meal-induced oxidative stress. Reduced insulin signaling in the brain appears to extend lifespan in various species. It is suggested that the insulin-signaling cascade in the brain could mediate this effect by being sensitive to peripheral nutrients. 


\title{
Dissociation of the insulin receptor and caveolin-1 complex by ganglioside GM3 in the state of insulin resistance
}

\author{
Kabayama K, Sato T, Saito K, Loberto N, Prinetti A, Sonnino S, Kinjo M, Igarashi Y, Inokuchi J \\ Division of Glycopathology, Institute of Molecular Biomembranes and Glycobiology, Tohoku Pharmaceutical \\ University, Sendai, Miyagi, Japan
}

Proc Natl Acad Sci USA 2007;104:13678-13683

Background: Membrane microdomains (lipid rafts) are now recognized as critical for proper compartmentalization of insulin signaling. The authors previously demonstrated that, in adipocytes in a state of $\mathrm{TNF} \alpha$-induced insulin resistance, the inhibition of insulin metabolic signaling and the elimination of insulin receptors from the caveolae microdomains were associated with an accumulation of the ganglioside GM3.

Methods: To gain insight into the molecular mechanisms behind interactions of insulin receptors, caveolin-1 (Cav1), and GM3 in adipocytes, the authors performed immunoprecipitations, cross-linking studies of insulin receptors and GM3, and live cell studies using total internal reflection fluorescence microscopy and fluorescence recovery after photobleaching techniques.

Results: The author found that (i) insulin receptors form complexes with Cav1 and GM3 independently; (ii) in GM3-enriched membranes the mobility of insulin receptors is increased by dissociation of the insulin receptors-Cav1 interaction, and (iii) the lysine residue localized just above the transmembrane domain of the insulin receptors $\beta$-subunit is essential for the interaction of insulin receptors with GM3. Conclusion(s): Because insulin metabolic signal transduction in adipocytes is known to be critically dependent on caveolae, the authors propose a pathological feature of insulin resistance in adipocytes caused by dissociation of the insulin receptors-Cav1 complex by the interactions of insulin receptors with GM3 in microdomains.

\section{Association of a homozygous nonsense caveolin-1 mutation with Berardinelli-Seip congenital lipodystrophy}

Kim CA, Delepine M, Boutet E, El Mourabit H, Le Lay S, Meier M, Nemani M, Bridel E, Leite CC, Bertola DR, Semple RK, O'Rahilly S, Dugail I, Capeau J, Lathrop M, Magre J

Department of Pediatrics, Instituto da Criança, University of Sao Paulo, Sao Paulo, Brazil

J Clin Endocrinol Metab 2008;93:1129-1134

Background: Berardinelli-Seip congenital lipodystrophy (BSCL) is a rare recessive disease characterized by near absence of adipose tissue, resulting in severe dyslipidemia and insulin resistance. In most reported cases, BSCL is due to alterations in either seipin, of unknown function, or 1-acylglycerol-3-phosphate acyltransferase- $\beta$ (AGPAT2), which catalyzes the formation of phosphatidic acid.

Objective: The authors sought to determine the genetic origin of the unexplained cases of BSCL. They thus sequenced CAV1, encoding caveolin-1, as a candidate gene involved in insulin signaling and lipid homeostasis. CAV1 is a key structural component of plasma membrane caveolae, and Cav1-deficient mice display progressive loss of adipose tissue and insulin resistance.

Methods: The authors undertook phenotyping studies and molecular screening of CAV1 in 4 patients with BSCL with no mutation in the genes encoding either seipin or AGPAT2.

Results: A homozygous nonsense mutation (p.Glu38X) was identified in CAV1 in a patient with BSCL born from a consanguineous union. This mutation affects both the $\alpha$ - and $\beta$-CAV1 isoforms and ablates CAV1 expression in skin fibroblasts. Detailed magnetic resonance imaging of the proband confirmed near total absence of both subcutaneous and visceral adipose tissue, with only vestigial amounts in the dorsal subcutaneous regions. In keeping with the lack of adipose tissue, the proband was also severely insulin-resistant and dyslipidemic. In addition, the proband had mild hypocalcemia likely due to vitamin D resistance.

Conclusion(s): These findings identify CAV1 as a new BSCL-related gene and support a critical role for caveolins in human adipocyte function.

Adipocyte membranes are known to play an important role in the function of these cells as illustrated by these two papers. Caveolae, a subset of membrane microdomains, are particularly abundant in 
adipocytes (they account for $20 \%$ of the plasma membrane area). It is one of the most important parts of the membrane as it plays the interface between hormonal signaling and endocytosis. Caveolins are the main component of caveolae. Three isoforms have been described, but although both CAV1 and CAV2 are expressed in adipocytes, only CAV1 has been shown to be both necessary and sufficient for caveolae formation. CAV1 is a key determinant of normal lipid homeostasis and insulin-regulated glucose uptake as the first paper illustrates. It shows that interactions between CAV1 and insulin receptor are essential for insulin signaling in adipocytes and that this interaction could be dissociated by other components of the membrane leading to altered insulin signaling. The case report of the second article furthermore establishes the critical importance of caveolae and CAV1 in normal adipocyte function in humans. Two genes have been reported to be associated with the Berardinelli-Seip syndrome or congenital lipoatrophy: BSCL2 encoding the seipin, a protein implicated in adiposomes synthesis, and BSCL1 encoding an enzyme (AGAPT 2) that catalyses phosphatidic acid, a key intermediate step in the synthesis of triglycerides. This article shows that a nonsense mutation in CAV1 leads to a phenotype of total lipoatrophy. Indeed, it provides compelling evidence for the critical requirement of caveolae for normal adipose tissue development. A similar phenotype is obtained in humans corresponding to the loss of function of seipin, AGPAT2 or CAV1 although the three molecules show seemingly disparate cellular functions. This striking observation may indicate functional connections between them and provides new insight into the in vivo physiology of adipose tissue.

\title{
Important for clinical practice Type 2 diabetes
}

\section{Metabolic abnormalities underlying the different prediabetic phenotypes in obese adolescents}

\author{
Cali AMGa, Bonadonna RC ${ }^{b}$, Trombetta $\mathrm{M}^{\mathrm{b}}$, Weiss $\mathrm{R}^{\mathrm{a}}$, Caprio $\mathrm{S}^{\mathrm{a}}$ \\ aDepartment of Pediatrics, Yale University School of Medicine, New Haven, Conn., USA, and bepartment of \\ Biomedical and Surgical Sciences, Section of Endocrinology, Università di Verona and Azienda Ospedaliera di Verona, \\ Verona, Italy \\ J Clin Endocrinol Metab 2008;93:1767-1773
}

Objective: The aim of this study was to define the metabolic abnormalities underlying the prediabetic status of isolated impaired fasting glucose (IFG), isolated impaired glucose tolerance (IGT), and combined IFG/IGT in obese youth.

Methods: Hyperinsulinemic-euglycemic and hyperglycemic clamps were performed in 40 normal glucose tolerance (NGT), 17 IFG, 23 IGT, and 11 IFG/IGT obese adolescents. Percent fat (by dual-energy X-ray absorptiometry), age, gender and ethnicity were comparable among groups.

Results: Peripheral insulin sensitivity was similar between the IFG and NGT groups. In contrast, the IGT and IFG/IGT groups showed marked reductions in peripheral insulin sensitivity $(\mathrm{p}<0.002)$. Basal hepatic insulin resistance index (basal hepatic glucose production $\times$ fasting plasma insulin) was significantly increased in IFG, IGT, and IFG/IGT $(\mathrm{p}<0.009)$ compared with NGT. Glucose sensitivity of first-phase insulin secretion was progressively lower in IFG, IGT, and IFG/IGT compared with NGT. Glucose sensitivity of second-phase secretion showed a statistically significant defect only in the IFG/IGT group. In a multivariate regression analysis, the glucose sensitivity of first-phase secretion and the basal insulin secretion rate were significant independent predictors of FPG (total $\mathrm{r}^{2}=25.9 \%$ ).

Conclusion(s): IFG, in obese adolescents, is linked primarily to alterations in glucose sensitivity of firstphase insulin secretion and liver insulin sensitivity. The IGT group is affected by a more severe degree of peripheral insulin resistance and reduction in first-phase secretion. IFG/IGT is hallmarked by a profound insulin resistance and by a new additional defect in second-phase insulin secretion.

Several papers have already evaluated the different alterations in glucose metabolism regulation associated with both IFG and/or IGT in adults [5-7] but this has not been studied in children in whom type 2 diabetes appears to evolve differently. The present paper is based on the gold standard techniques for the measurement of insulin sensitivity and insulin secretion together with a refined way of 
calculating first-phase insulin release. As could be anticipated, IFG is related to hepatic insulin resistance and alteration of the first-phase insulin release while IGT involves insulin resistance. Combined IFG/IGT encompasses a more severe insulin resistance and a defect in both phases of insulin secretion. The authors propose that one way of maintaining fasting glucose levels within the normal range is an increased basal glucose clearance, defined as the ratio of basal glucose turnover rate to fasting plasma glucose, reflecting a better glucose effectiveness, which is the ability of the glucose itself to be taken up by insulin-sensitive organs (mainly muscle and liver) in the fasting sate. The exact mechanisms of these compensatory pathways remain poorly elucidated.

Longitudinal follow-up of such children with different disorders of glucose tolerance will help determine how they progress towards type 2 diabetes and which of either defect between insulin secretion and insulin resistance is predictive of the overt disease.

\section{Childhood predictors of young-onset type 2 diabetes}

Franks PW, Hanson RL, Knowler WC, Moffett C, Enos G, Infante AM, Krakoff J, Looker HC Diabetes Epidemiology and Clinical Research Section, National Institute of Diabetes and Digestive and Kidney Diseases, National Institutes of Health, Phoenix, Ariz., USA

Diabetes 2007; 56:2964-2972

Background: Optimal prevention of young-onset type 2 diabetes requires identification of the early-life modifiable risk factors. The study aimed to do this using longitudinal data in 1,604 five- to 19-year-old initially nondiabetic American Indians.

Methods: For type 2 diabetes (T2D) prediction, an index was constructed (zMS) comprising commonly measured metabolic, anthropometric, and vascular traits (i.e., fasting and 2-hour glucose, A1C, BMI, waist circumference, fasting insulin, HDL cholesterol, triglycerides, and blood pressures) and compared the predictive power for each feature against zMS.

Results: In separate Cox proportional hazard models, adjusted for age, sex, and ethnicity, zMS and each of its component risk factors were associated with incident T2D. Stepwise proportional hazards models selected fasting glucose, 2-hour glucose, HDL cholesterol, and BMI as independent diabetes predictors; individually, these were weaker predictors than zMS $(\mathrm{p}<0.01)$. However, a parsimonious summary score combining only these variables had predictive power similar to that of zMS $(p=0.33)$. Although intrauterine diabetes exposure or parental history of young-onset diabetes increased a child's absolute risk of developing diabetes, the magnitude of the diabetes-risk relationships for zMS and the parsimonious score were similar regardless of familial risk factors.

Conclusion(s): The relative value of the features of the metabolic syndrome in childhood was assessed for the prediction of subsequent T2D. Data suggest that strategies targeting obesity, dysregulated glucose homeostasis, and low HDL cholesterol during childhood and adolescence may have the most success in preventing diabetes.

Based on a prospective study that, probably, can only be performed in Pima Indians, given the very high and unusual rate of T2D ( 1 of 2 adult individuals), the data presented here outline risk factors for T2D in children and point therefore to the very children to whom preventive intervention could be targeted. On the one hand, one can argue that Pima Indians are a very special population; on the other hand, most of the observations made in adults with respect to the natural history of T2D happened to hold true in other ethnicities. In the younger age group, waist circumference appears to be a better predictor than BMI itself underlining that BMI is not always the best surrogate measure for fatness in childhood. 2-Hour glucose contributes to the risk, although it does not remain significant in the multivariate analysis. In adolescents, 2-hour glucose was the best predictor. In the entire population, the best predictors were 2-hour glucose, waist circumference and BMI as well as HDL-cholesterol. Parental history of T2D increased the risk in the offspring as indicated by a 3-fold increase in incidence. Intrauterine exposure to maternal diabetes is associated with a 5 -fold increase in risk. Altogether, these results show that it is feasible to identify children at increased risk of T2D based on common parameters assessed under clinical practice. 


\section{Metabolic syndrome in childhood predicts adult metabolic syndrome and type $\mathbf{2}$ diabetes mellitus $\mathbf{2 5}$ to $\mathbf{3 0}$ years later}

Morrison JA, Friedman LA, Wang P, Glueck CJ

Division of Cardiology, Cincinnati Children's Hospital Medical Center, Cincinnati, Ohio, USA

J Pediatr 2008;152:201-206

Background: The metabolic syndrome (MS) is non-universally defined in children by the clustering of at least three of five of the most serious risk factors for cardiovascular diseases. The tracking of MS from childhood to adults is rarely reported. The authors prospectively assessed the association of the MS in childhood with adult MS and type 2 diabetes mellitus (T2DM) 25-30 years later.

Methods: Data from the National Heart Lung and Blood Institute Lipid Research Clinics (LRC) Princeton Prevalence Study (1973-1976) and the Princeton Follow-up Study (PFS, 2000-2004) were used. Body mass index $\left(\mathrm{BMI}=\mathrm{kg} / \mathrm{m}^{2}\right)$ was used as the obesity measure in childhood because waist circumference was not measured at the LRC. The adult T2DM status of participants and their parents was obtained by participant report or a fasting blood glucose of $\geq 126 \mathrm{mg} / \mathrm{dl}$. A logistic analysis for clustered samples was used to predict adult MS and T2DM, taking into account sibling correlations in the cohort. Pediatric MS, age at PFS, sex, race, change in BMI percentile, parental history of diabetes, and the interaction of pediatric MS and parental diabetes were explanatory variables.

Results: Ages ranged from 5 to 19 years in the LRC and from 30 to 48 years in the PFS. Pediatric MS, parental diabetes, age at follow-up, and change in age-specific BMI percentile were significant predictors of MS in adulthood, and pediatric MS, age at follow-up, black race, and parental diabetes were significant predictors of T2DM.

Conclusion(s): Evaluating 5- to 19-year-old children for MS and family history of diabetes could identify children at increased risk of adult MS and T2DM, allowing prospective primary prevention of these outcomes.

We have already discussed the meaning of the MS in children in previous issues of the Yearbook in Pediatric Endocrinology [8, 9]. In this cohort, the prevalence of T2DM was 5.4\% in children and $15.6 \%$ in the same individuals grown to adulthood. Children with MS were more likely to develop T2DM when adults. Pediatric MS and a parental history of T2DM were the stronger predictors of adult T2DM, with probably a synergistic effect. Prevalence of MS was $4 \%$ in children and $27.2 \%$ in adults, reflecting the marked increase in BMI. MS in adults was strongly predicted by MS in children and an increase in weight as a $10 \%$ increase in BMI increases the risk of adult MS by $25 \%$. These observations highlight the central role of obesity and associated insulin resistance. The same authors have also published more detailed predictive values of the features of pediatric MS for adult MS [10].

A parental history of T2DM was associated with a higher BMI in both children and adults illustrating the contribution of T2DM itself in the acceleration of the disease. Indeed, the process involves transmission of susceptibility genes for the disease. It has been reported that the offspring of parents with MS had lower insulin sensitivity [11]. Moreover, it has also been demonstrated that maternal diabetes during fetal development favors both obesity and an earlier age at onset of T2DM [12], the latter being promoted by the programming of impaired insulin secretion [13].

\section{The metabolic syndrome and non-alcoholic steatohepatitis (NASH)}

\section{Metabolic syndrome and liver histology in pediatric non-alcoholic steatohepatitis}

Manco M, Marcellini M, Devito R, Comparcola D, Sartorelli MR, Nobili V

Liver Unit, Pathology Department, 'Bambino Gesu' Children's Hospital and Research Institute, Rome, Italy

Int J Obes (Lond) 2008;32:381-387

Background: The aim was to estimate prevalence of metabolic syndrome (MS), obesity and comorbidities in a cohort of 120 children (3-18 years) with biopsy-proven non-alcoholic fatty liver disease or nonalcoholic steatohepatitis (NASH) and to evaluate correlations between clinical or biochemical variables and liver histology. 
Methods: MS was diagnosed according to the adapted National Cholesterol Education Program criteria. Homeostatic model assessment of insulin resistance (HOMA-IR), quantitative insulin-sensitivity check index (QUICKI), and ISI composite, insulin secretion (insulin response $30 \mathrm{~min}$ after a glucose load; HOMA- $\beta$ cell; insulinogenic index) were all estimated. BMI z-score and total body fat (dual-energy Xray absorptiometry) were evaluated as indexes of obesity.

Results: MS was diagnosed in $66 \%$ of children. About $92 \%$ had weight above the 85 th percentile, of which $42 \%$ were obese with weight above 97 th percentile. The prevalence of hypertriglyceridemia was $63 \%$, low HDL cholesterol $45 \%$, hypertension $40 \%$, and impaired glucose tolerance $10 \%$. Levels of aminotransferases were higher as the number of comorbidities increased, the highest values being found in subjects with MS $(\mathrm{p} \leq 0.05)$. The prevalence of a grade of steatosis of $\geq 2(\mathrm{p}=0.05)$ and fibrosis $(\mathrm{p} \leq 0.01)$ was higher in subjects with MS. Histology was associated significantly with higher values of a number of clinical and biochemical parameters (steatosis $\geq 2$ with BMI z-score $(p=0.04)$, fasting insulin ( $\mathrm{p}=0.02)$, HOMA-IR $(\mathrm{p}=0.03), \beta$-cell secretion $(\mathrm{p}=0.04)$; necro-inflammation with BMI $\mathrm{z}$ score $(\mathrm{p}=0.007)$, glucose $(\mathrm{p} \leq 0.0001)$, cholesterol $(\mathrm{p} \leq 0.04)$ and white blood cells $(\mathrm{p}=0.025)$; fibrosis with body weight $(\mathrm{p}=0.05)$, BMI $\mathrm{z}$-score $(\mathrm{p}=0.03)$, cholesterol $(\mathrm{p}=0.05)$, triglycerides $(\mathrm{p}=0.05)$, fasting insulin $(\mathrm{p} \leq 0.0001)$ and mean values of the hormone at the OGTT $(\mathrm{p}=0.03)$, HOMA-IR $(\mathrm{p} \leq 0.0001))$.

Conclusion(s): The presence of MS or clinical and biochemical variables associated with the syndrome seem to be strictly related to histological features of NASH in pediatric fatty liver disease. Thus, routine liver biopsy should be encouraged in these children.

The association between MS and NASH is not new but has not been extensively documented in children and this paper is based on a group of children with results on liver histology. One paper reported that a diagnosis of NASH may be suspected in one fourth of obese children [14]. Roughly, MS is observed on $3-5 \%$ of healthy children, in one fourth to one third of obese children, and here it is shown that about two thirds of the children affected by NASH can be simultaneously diagnosed with the full MS. The levels of AST and ALT were correlated with the number of comorbidities included in the definition of MS and it was no big surprise that obesity came first. Conversely, children with MS showed more severe hepatic alterations and more frequently liver fibrosis. Two observations are worth being noted:

- In the study population, $2 \%$ of the children were diagnosed with type 2 diabetes by OGTT.

- In patients with liver fibrosis, the glucose disposition index was shifted to the left, showing that not only these children are more insulin-resistant but also that their $\beta$-cell function was not able to fully compensate for IR. The reason for this impaired insulin secretion is not clear.

Similar to the previous paper, the present one underlies the complex network between IR, MS, HTA and NASH, the intricacy of which must be more precisely documented in children before proposing various interventions. One point is clear: all these comorbidities are strongly obesity-driven.

\section{Metabolic disorders and primary hypertension}

\section{Metabolic abnormalities, insulin resistance, and metabolic syndrome in children with primary hypertension}

Litwin M, Sladowska J, Antoniewicz J, Niemirska A, Wierzbicka A, Daszkowska J, Wawer ZT, Janas R, Grenda R Department of Nephrology, Kidney Transplantation, and Arterial Hypertension, Children's Memorial Health Institute, Warsaw, Poland

Am J Hypertens 2007;20:875-882

Background: The aim was to study the prevalence of metabolic abnormalities and the metabolic syndrome (MS) and its relationship to target-organ damage in children with primary hypertension (PH).

Methods: Patients included 113 children with untreated $\mathrm{PH}$ at a mean age of 14.6 (range 5-18) years. The control group consisted of 134 healthy children at a mean age of 13.5 (range 5-20) years. The study is based on a cross-sectional assessment of anthropometric and biochemical cardiovascular risk factors, homeostatic metabolic assessment (HOMA-IR), the insulin sensitivity index (ISI) and adiponectin. 
Results: MS, as defined by classic criteria, was present in 4 of 134 (3\%) controls versus 23 of $113(20.4 \%)$ patients $(\mathrm{p}=0.0001)$, but when PH was not taken as a criterion of MS, MS was diagnosed in $6.2 \%$ of patients (not significant). Left-ventricular hypertrophy (LVH) was found in 46 of 113 patients $(40.7 \%)$, and severe LVH was found in 14 of 113 patients $(12.5 \%)$. Patients with LVH had a greater body mass index, greater waist-to-hip ratio, and greater number of parameters of MS (overall $\mathrm{p}<0.05$ ). Carotid (cIMT) and femoral superficial artery intima-media thicknesses correlated positively with HOMA-IR and negatively with ISI and serum adiponectin $(\mathrm{p}<0.05)$. The main predictor for cIMT was adiponectin $\left(\mathrm{R}^{2}=0.178, \beta=-0.466, \mathrm{p}=0.002\right)$. LVH was predicted $\left(\mathrm{R}^{2}=0.332\right)$ by the body mass index standard deviation score $(\beta=0.551, p=0.005)$ and HOMA-IR $(\beta=0.380, p=0.04)$.

Conclusion(s): MS, as defined by classic criteria, was diagnosed in $20 \%$ of children with PH, but when PH was not used as a criterion, MS was present in $6.2 \%$ of patients. Regardless of the definition of MS, the applied markers of MS and insulin resistance were the main predictors of target-organ damage.

The question of what comes first - the egg or the hen - has been raised about MS and HTA since the early 1980s. However, this paper is the first to document this question in children. In the present study, all parameters of MS were more elevated in children with HTA than in controls. It shows that MS is much more frequent in children with HTA and, even though BP is not included in the definition of MS, the latter remain clearly more frequent. Children with primary HTA, who concomitantly are classified as having MS, show more severe cardiac features than the other children with HTA. Moreover, early vascular damage shows a strong negative correlation with both adiponectin and markers of IR. It appears that most, if not all, of these changes were related to obesity; however, children with obesity and HTA show more severe metabolic changes that children with obesity alone. This paper is one among numerous others that should draw our attention to the care of obese children with respect to the metabolic and cardiovascular consequences.

\section{Clinical trial}

\section{Interleukin-1-receptor antagonist in type 2 diabetes mellitus}

Larsen CM, Faulenbach M, Vaag A, Volund A, Ehses JA, Seifert B, Mandrup-Poulsen T, Donath MY Steno Diabetes Center, Gentofte, Denmark

N Engl J Med 2007;356:1517-1526

Background: The expression of interleukin-1-receptor antagonist is reduced in the pancreatic islets of patients with type 2 diabetes mellitus, and high glucose concentrations induce the production of interleukin-1 $\beta$ in human pancreatic $\beta$ cells, leading to impaired insulin secretion, decreased cell proliferation, and apoptosis.

Methods: In this double-blind, parallel-group trial involving 70 patients with type 2 diabetes, the authors randomly assigned 34 patients to receive $100 \mathrm{mg}$ anakinra (a recombinant human interleukin-1-receptor antagonist) subcutaneously once daily for 13 weeks and 36 patients to receive placebo. At baseline and at 13 weeks, all patients underwent an oral glucose-tolerance test, followed by an intravenous bolus of $0.3 \mathrm{~g}$ of glucose $/ \mathrm{kg}$ body weight, $0.5 \mathrm{mg}$ glucagon, and $5 \mathrm{~g}$ arginine. In addition, 35 patients underwent a hyperinsulinemic-euglycemic clamp study. The primary end point was a change in the level of glycated hemoglobin, and secondary end points were changes in $\beta$-cell function, insulin sensitivity, and inflammatory markers.

Results: At 13 weeks, in the anakinra group, the glycated hemoglobin level was 0.46 percentage points lower than in the placebo group $(\mathrm{p}=0.03)$; C-peptide secretion was enhanced $(\mathrm{p}=0.05)$, and there were reductions in the ratio of proinsulin to insulin $(\mathrm{p}=0.005)$ and in levels of interleukin-6 $(\mathrm{p}<0.001)$ and C-reactive protein $(\mathrm{p}=0.002)$. Insulin resistance, insulin-regulated gene expression in skeletal muscle, serum adipokine levels, and the body-mass index were similar in the two study groups. Symptomatic hypoglycemia was not observed, and there were no apparent drug-related serious adverse events.

Conclusion(s): The blockade of interleukin-1 with anakinra improved glucose control and $\beta$-cell secretory function and reduced markers of systemic inflammation. 
The reason this paper is here is that a very novel way of treating type 2 diabetes has been tested. So far drugs targeting inflammation in the islets have been tested in type 1 diabetes. However, it appeared that proinflammatory cytokines play a role in type 2 diabetes by inducing apoptosis and accelerated destruction of the $\beta$-cells. Data on observations of the pancreas of patients with type 2 diabetes suggest intra-islet production of inflammatory cytokines. IL-1-receptor antagonist is a natural inhibitor of IL-1 binding to its receptor and protects human $\beta$-cells from glucose toxicity and its expression is reduced in type 2 diabetes. By intervening on this pathway, the authors report a modest but clear effect on blood glucose control explained by improvement in insulin secretion with no change in insulin resistance or BMI. The lack of correlation between the reduction of glycated hemoglobin and circulating inflammatory markers does not argue in favor of the role of systemic inflammation. Indeed, this trial was of short duration and further data are needed prior to expansion of this treatment to more general practice.

\title{
New mechanisms Mitochondria and oxidation
}

\section{Impaired mitochondrial substrate oxidation in muscle of insulin-resistant offspring of type 2 diabetic patients}

\author{
Befroy DE, Petersen KF, Dufour S, Mason GF, de Graaf RA, Rothman DL, Shulman GI \\ Department of Internal Medicine, Yale University School of Medicine, New Haven, Conn., USA \\ Diabetes 2007; 56:1376-1381
}

Background: Insulin resistance is the best predictor for the development of diabetes in the offspring of type 2 diabetic patients, but the mechanism responsible for it remains unknown. Recent studies have demonstrated increased intramyocellular lipid, decreased mitochondrial ATP synthesis, and decreased mitochondrial density in the muscle of lean, insulin-resistant offspring of type 2 diabetic patients. These data suggest an important role for mitochondrial dysfunction in the pathogenesis of type 2 diabetes.

Methods and Subjects: To further explore this hypothesis, the authors assessed rates of substrate oxidation in the muscle of these same individuals using ${ }^{13} \mathrm{C}$ magnetic resonance spectroscopy (MRS). Young, lean, insulin-resistant offspring of type 2 diabetic patients and insulin-sensitive control subjects underwent ${ }^{13} \mathrm{C}$ MRS studies to noninvasively assess rates of substrate oxidation in muscle by monitoring the incorporation of ${ }^{13} \mathrm{C}$ label into ${ }^{4} \mathrm{C}$-glutamate during a labeled acetate infusion.

Results: Using this approach, the authors found that rates of muscle mitochondrial substrate oxidation were decreased by $30 \%$ in lean, insulin-resistant offspring $\left(59.8 \pm 5.1 \mathrm{nmol} \times \mathrm{g}^{-1} \times \mathrm{min}^{-1}, \mathrm{p}=0.02\right)$ compared with insulin-sensitive control subjects $\left(96.1 \pm 16.3 \mathrm{nmol} \times \mathrm{g}^{-1} \times \mathrm{min}^{-1}\right)$.

Conclusion(s): These data support the hypothesis that insulin resistance in skeletal muscle of insulin-resistant offspring is associated with dysregulation of intramyocellular fatty acid metabolism, possibly because of an inherited defect in the activity of mitochondrial oxidative phosphorylation.

Impaired mitochondrial function has been suspected for years to play an important role in the pathophysiology of type 2 diabetes (T2D). This association was first based on important but indirect data reporting that insulin resistance is associated with a decreased mitochondrial number in muscle. This lower density has relevant functional consequences by driving lower ATP synthesis. The authors of this paper have contributed to these observations. But two questions remain open: is this decreased function a consequence or a cause of insulin resistance and then, are these abnormalities present at an early stage and, if so, do they play an important role in the transition from isolated IR to type two diabetes? Here the authors decided to focus on young offspring of type 2 diabetic patients showing insulin resistance without glucose tolerance problems. The subjects were selected with no other risk factors of type two diabetes (no overweight, no smoking, etc.) in order to exclude all confounding factors that could participate in insulin resistance or affect mitochondrial function. Noninvasive methods were used coupling Spectro-MRI and labeled substrates to measure the rate of lipid oxidation via the tricarboxylic acid cycle. The authors reported direct evidence of lipid accumulation in muscle associated with impaired mitochondrial function. This report supports the idea that impaired 
mitochondrial function occurs at an early stage of insulin resistance in subjects at risk of type 2 diabetes. The accumulation of lipids has relevant consequences since it favors diacylglycerol accumulation leading to alteration of insulin signaling in muscle. The molecular and/or genetic cause of this dysfunction is still unknown. In these subjects, no decreased expression of known genes involved in mitochondrial oxidation and biogenesis were found, suggesting that our knowledge on these mechanisms is still incomplete. A final remark is that the methods used by the authors are of utmost interest since they are not invasive and could be translated into pediatric clinical investigation.

\title{
Mitochondrial dysfunction results from oxidative stress in the skeletal muscle of diet-induced insulin-resistant mice
}

\author{
Bonnard C, Durand A, Peyrol S, Chanseaume E, Chauvin MA, Morio B, Vidal H, Rieusset J \\ INSERM, U870, IFR62, Oullins, France \\ J Clin Invest 2008;118:789-800
}

Background: Mitochondrial dysfunction in skeletal muscle has been implicated in the development of type 2 diabetes. However, whether these changes are a cause or a consequence of insulin resistance is not clear. Methods: The authors investigated the structure and function of muscle mitochondria during the development of insulin resistance and progression to diabetes in mice fed a high-fat, high-sucrose diet.

Results: Although 1 month of high-fat, high-sucrose diet feeding was sufficient to induce glucose intolerance, mice showed no evidence of mitochondrial dysfunction at this stage. However, an extended diet intervention induced a diabetic state in which the authors observed altered mitochondrial biogenesis, structure, and function in muscle tissue. The authors assessed the role of oxidative stress in the development of these mitochondrial abnormalities and found that diet-induced diabetic mice had an increase in ROS production in skeletal muscle. In addition, ROS production was associated with mitochondrial alterations in the muscle of hyperglycemic streptozotocin-treated mice, and normalization of glycemia or antioxidant treatment decreased muscle ROS production and restored mitochondrial integrity.

Conclusion(s): Glucose- or lipid-induced ROS production resulted in mitochondrial alterations in muscle cells in vitro, and these effects were blocked by antioxidant treatment. These data suggest that mitochondrial alterations do not precede the onset of insulin resistance and result from increased ROS production in muscle in diet-induced diabetic mice.

This article illustrates and clarifies most of our knowledge on mitochondrial dysfunction and type 2 diabetes. The aim was to answer two important questions: does mitochondrial dysfunction appear prior to insulin resistance and what causes this dysfunction? The authors used different animal models of diabetes to answer these questions and further explored the role of oxidative stress in cultured myotubes. After a high-fat diet, mice demonstrate mitochondrial dysfunction that mimics that reported in humans. Insulin resistance and diabetes are associated with altered mitochondrial density, structure and function. First, the authors report that mitochondrial dysfunction can appear at an early stage of type 2 diabetes and it does not enhance insulin resistance; mitochondrial dysfunction is not present before diabetes and furthermore can be detected in diabetes not associated with insulin resistance. Second, they assess that oxidative stress and ROS play an important role in these defects. Third, they show that excessive ROS production is not a consequence of insulin resistance but of lipid excess and is further increased by hyperglycemia. This article shows that mitochondrial dysfunction reported in type 2 diabetic patients is not the initial event that triggers decreased oxidative capacity and lipid accumulation and then inhibition of the insulin pathway but rather is a complication of hyperlipidemia, which induces ROS production in skeletal muscle. The authors suggest that increased oxidative stress in muscle could be a unifying mechanism promoting mitochondrial alterations, lipid accumulation and insulin resistance. It could also be a more general mechanism in type 2 diabetes by contributing to the two prominent features of type 2 diabetes: insulin resistance and pancreatic $\beta$ cell dysfunction. As such, it represents a suitable target for therapeutic intervention. 


\title{
Mitochondrial dysfunction due to long-chain Acyl-CoA dehydrogenase deficiency causes hepatic steatosis and hepatic insulin resistance
}

Zhang D, Liu ZX, Choi CS, Tian L, Kibbey R, Dong J, Cline GW, Wood PA, Shulman GI

Howard Hughes Medical Institute, Yale University School of Medicine, New Haven, Conn., USA

Proc Natl Acad Sci USA 2007;104:17075-17080

Background: Alterations in mitochondrial function have been implicated in the pathogenesis of insulin resistance and type 2 diabetes. However, it is unclear whether the reduced mitochondrial function is a primary or acquired defect in this process.

Methods: To determine whether primary defects in mitochondrial $\beta$-oxidation can cause insulin resistance, the authors studied mice with a deficiency in long-chain acyl-CoA dehydrogenase (LCAD), a key enzyme in mitochondrial fatty acid oxidation.

Results: The authors show that LCAD knockout mice develop hepatic steatosis, which is associated with hepatic insulin resistance, as reflected by reduced insulin suppression of hepatic glucose production during a hyperinsulinemic-euglycemic clamp. The defects in insulin action were associated with an approximately $40 \%$ reduction in insulin-stimulated insulin receptor substrate-2-associated phosphatidylinositol 3-kinase activity and an approximately 50\% decrease in Akt2 activation. These changes were associated with increased PKC $\varepsilon$ activity and an aberrant 4-fold increase in diacylglycerol content after insulin stimulation. The increase in diacylglycerol concentration was found to be caused by de novo synthesis of diacylglycerol from medium-chain acyl-CoA after insulin stimulation.

Conclusion(s): These data demonstrate that primary defects in mitochondrial fatty acid oxidation capacity can lead to diacylglycerol accumulation, PKC $\varepsilon$ activation, and hepatic insulin resistance.

\begin{abstract}
Altered mitochondrial function can play an important role in the development of hepatic insulin resistance. The liver has a strategic position and coordinates nutrient flux between organs. It regulates the export and import of triglycerides and cholesterol and can then in turn promote changes in body composition in case of insulin resistance. Here, the authors developed a mouse model demonstrating a mitochondrial defect in lipid oxidation. Their results show that decreased $\beta$-oxidation participates in hepatic insulin resistance and favors the appearance of steatosis. In the fed state, under insulin stimulation, exogenous fatty acid will be used to produce diacylglycerol, a substrate that can alter insulin signaling when its intracellular concentration is increased. This pool of diacylglycerol will be used to increase postprandial triglyceride synthesis. This paper shows that alteration of $\beta$-oxidation in the liver will have consequences on whole body nutrient flux. It will favor de novo triglyceride synthesis and export leading to an increased fat mass. It will also promote the development of atherogenic dyslipidemia. This study illustrates the central role played by the liver in abnormalities associated with insulin resistance. Interestingly, here the reduced mitochondrial lipid oxidation has no effect on muscle insulin sensitivity strengthening the idea that mitochondrial dysfunction does not occur prior to insulin resistance.
\end{abstract}

\section{Fat oxidation}

\section{Continuous fat oxidation in acetyl-CoA carboxylase-2 knockout mice increases total energy expenditure, reduces fat mass, and improves insulin sensitivity}

Choi CS, Savage DB, Abu-Elheiga L, Liu ZX, Kim S, Kulkarni A, Distefano A, Hwang YJ, Reznick RM, Codella R, Zhang D, Cline GW, Wakil SJ, Shulman Gl

Department of Internal Medicine, Yale University School of Medicine, New Haven, Conn., USA

Proc Natl Acad Sci USA 2007;104:16480-1648

Background: Acetyl-CoA carboxylase (ACC)-2 is a key regulator of mitochondrial fat oxidation. Methods: To examine the impact of ACC2 deletion on whole-body energy metabolism, we measured changes in substrate oxidation and total energy expenditure in $\mathrm{Acc}^{-/-}$and WT control mice fed either regular or high-fat diets. To determine insulin action in vivo, the authors also measured whole-body 
insulin-stimulated liver and muscle glucose metabolism during a hyperinsulinemic-euglycemic clamp in $\mathrm{Acc}^{-/-}$and WT control mice fed a high-fat diet.

Results: Contrary to previous studies that have suggested that increased fat oxidation might result in lower glucose oxidation, both fat and carbohydrate oxidation were simultaneously increased in Acc $2^{-I-}$ mice. This increase in both fat and carbohydrate oxidation resulted in an increase in total energy expenditure, reductions in fat and lean body mass and prevention from diet-induced obesity. Furthermore, Acc2 $2^{-/-}$mice were protected from fat-induced peripheral and hepatic insulin resistance. These improvements in insulin-stimulated glucose metabolism were associated with reduced diacylglycerol content in muscle and liver, decreased PKC activity in muscle and PKC $\varepsilon$ activity in the liver, and increased insulin-stimulated Akt2 activity in these tissues.

Conclusion(s): Taken together with previous work demonstrating that Acc $2^{-/-}$mice have a normal lifespan, these data suggest that Acc 2 inhibition is a viable therapeutic option for the treatment of obesity and type 2 diabetes.

Rather than a signaling disease, insulin resistance and type 2 diabetes seem to be nutrient-flux disorders. Many data suggest that changing whole body nutrient flux plays a large role in the pathogenesis of type 2 diabetes. If these changes can accelerate the process, can other changes in opposite directions protect against diabetes? Here, the authors describe the metabolic consequences of increasing lipid oxidation by inhibiting a key enzyme acetyl-CoA carboxylase. This enzyme plays an important role in enhancing malonyl-CoA synthesis and then inhibiting lipid oxidation and promoting lipid synthesis. On the one hand, one would think that this inhibition could protect against dietinduced diabetes by decreasing lipid accumulation, but on the other hand under Randle's hypothesis fat oxidation and carbohydrate oxidation are thought to be mutually inhibitory. Increased fat oxidation could then promote insulin resistance and its deleterious metabolic consequences by changing glucose flux. Here, the artificial increase in fat oxidation enhances energy expenditure and has no effect on glucose flux. It globally increases whole body insulin sensitivity and glucose uptake to muscle and liver. The mice demonstrated an increased influx of triglycerides from adipose tissue to muscle and to the liver for oxidation. Changing metabolic flux and their utilization in oxidative cells can then have beneficial effects. Increased lipid accumulation and its consequences on insulin signaling in muscle seem to be more deleterious than increased lipid oxidation in the pathogenesis of type 2 diabetes. Therapeutic interventions targeting enzymes which regulate nutrient flux could therefore be successful in the treatment of type 2 diabetes.

\section{Impaired fat oxidation after a single high-fat meal in insulin-sensitive nondiabetic individuals with a family history of type 2 diabetes}

Heilbronn LK, Gregersen S, Shirkhedkar D, Hu D, Campbell LV

Diabetes and Obesity Program, Garvan Institute of Medical Research, N.S.W., Australia

Diabetes 2007:56:2046-2053

Background: Individuals with insulin resistance and type 2 diabetes have an impaired ability to switch appropriately between carbohydrate and fatty acid oxidation. However, whether this is a cause or consequence of insulin resistance is unclear, and the mechanism(s) involved in this response is not completely elucidated.

Methods and Subjects: Whole-body fat oxidation and transcriptional regulation of genes involved in lipid metabolism in skeletal muscle were measured after a prolonged fast and after consumption of either high-fat $(76 \%)$ or high-carbohydrate $(76 \%)$ meals in individuals with no family history of type 2 diabetes (control, $\mathrm{n}=8$ ) and in age- and fatness-matched individuals with a strong family history of type 2 diabetes $(\mathrm{n}=9)$. Vastus lateralis muscle biopsies were performed before and $3 \mathrm{~h}$ after each meal.

Results: Insulin sensitivity and fasting measures of fat oxidation were not different between groups. However, subjects with a family history of type 2 diabetes had an impaired ability to increase fatty acid oxidation in response to the high-fat meal $(\mathrm{p}<0.05)$. This was related to impaired activation of genes involved in lipid metabolism, including those for peroxisome proliferator-activated receptor coactivator- $1 \alpha(\mathrm{PGC} 1 \alpha)$ and fatty acid translocase (FAT/CD36; $\mathrm{p}<0.05)$. Of interest, adiponectin receptor-1 expression decreased by $23 \%$ after the high-fat meal in both groups, but it was not changed after the high-carbohydrate meal. 
Conclusion(s): An impaired ability to increase fatty acid oxidation precedes the development of insulin resistance in genetically susceptible individuals. PGC1 $\alpha$ and FAT/CD36 are likely candidates in mediating this response.

As nutrient flux is supposed to play an important role in metabolic disorders such as insulin resistance, type 2 diabetes or the metabolic syndrome, the authors explored nutrient utilization in subjects at a high risk of type 2 diabetes without overt insulin resistance. Metabolic inflexibility is a concept that has been advanced in the pathogenesis of insulin resistance and is seen as the impaired ability to switch appropriately between carbohydrates and fatty acid oxidation, which in turn would lead to increased fatty acid accumulation in muscle and then favor insulin resistance. Few studies deal with this phenomenon in the pathogenesis of type 2 diabetes or insulin resistance and ask whether it is a cause or a consequence of insulin resistance. In contrast to the previously commented paper, subjects at a high risk of type 2 diabetes failed to increase lipid oxidation after a high-fat meal associated with a global decrease in enzymes involved in lipid metabolism. As metabolic flexibility is highly variable between subjects, this paper shows that the intrinsic ability to change the oxidation level of the different nutrients modulates an individual's susceptibility to type 2 diabetes and insulin resistance. Promoting interventions to favor oxidation fluxes (such as physical activity) and fighting against situations leading to alterations in nutrient flux are of great interest. These interventions however must not be seen as just a way to modulate global energy expenditure but must be thought of as a way to modulate this flexibility.

\section{The concept of insulin resistance}

\section{The role of skeletal muscle insulin resistance in the pathogenesis of the metabolic syndrome}

Petersen KF, Dufour S, Savage DB, Bilz S, Solomon G, Yonemitsu S, Cline GW, Befroy D, Zemany L, Kahn BB, Papademetris X, Rothman DL, Shulman GI

Department of Internal Medicine, Yale University School of Medicine, New Haven, Conn., USA

Proc Natl Acad Sci USA 2007;104:12587-12594

Background: The authors examined the hypothesis that insulin resistance in skeletal muscle promotes the development of atherogenic dyslipidemia, associated with the metabolic syndrome, by altering the distribution pattern of postprandial energy storage.

Methods: Glycogen synthesis, hepatic de novo lipogenesis and hepatic triglyceride synthesis following ingestion of two high carbohydrate mixed meals was measured in young, lean, insulin-resistant subjects compared with a similar cohort of age-, weight-, body mass index- and activity-matched, insulin-sensitive, control subjects.

Results: Net muscle glycogen synthesis was reduced by approximately $60 \%$ in insulin-resistant subjects compared with control subjects. In contrast, hepatic de novo lipogenesis and hepatic triglyceride synthesis were both increased by $>2$-fold in the insulin-resistant subjects. These changes were associated with a $60 \%$ increase in plasma triglyceride concentrations and an approximately $20 \%$ reduction in plasma high-density lipoprotein concentrations but no differences in plasma concentrations of TNF- $\alpha$, IL-6, adiponectin, resistin, retinol-binding protein-4, or intra-abdominal fat volume.

Conclusion(s): These data demonstrate that, due to decreased muscle glycogen synthesis, insulin resistance in skeletal muscle can promote atherogenic dyslipidemia by changing the pattern of ingested carbohydrate away from skeletal muscle glycogen synthesis into hepatic de novo lipogenesis, resulting in an increase in plasma triglyceride concentrations and a reduction in plasma high-density lipoprotein concentrations. Furthermore, insulin resistance in these subjects was independent of changes in the plasma concentrations of TNF- $\alpha$, IL-6, high-molecular-weight adiponectin, resistin, retinol-binding protein-4, or intra-abdominal obesity, suggesting that these factors do not play a primary role in causing insulin resistance in the early stages of the metabolic syndrome.

For a long time insulin resistance has been considered a consequence of fat mass accumulation. Insulin resistance was then mostly seen as a consequence of obesity also driving the metabolic syndrome. 
Visceral fat mass and changes in adipokine secretion were thus studied to better understand the pathophysiology of the metabolic syndrome. But insulin resistance is a real disease that involves not only insulin signaling but also whole-body nutrient fluxes. This article is highly relevant from this point of view and is more a conceptual paper than a report of a (high-quality) clinical research. The authors hypothesized that insulin resistance alone promotes the metabolic syndrome. They studied highly selected subjects in whom the only abnormality was insulin resistance in order to exclude all other confounding factors. They show that muscular insulin resistance promotes the change in glucose flux and the conversion of ingested carbohydrates into hepatic de novo lipogenesis. This leads to increased plasma VLDL, the triglyceride part of which will be exchanged for HDL cholesterol under physiological conditions. This in turn will lead to atherogenic cholesterol-rich VLDL remnant particles and triglyceride-rich cholesterol depleted $\mathrm{HDL}$ that could undergo further modifications towards lower HDL-cholesterol and Apo-A1. This paper also shows that muscular insulin resistance promotes hepatic steatosis and that increased hepatic de novo lipogenesis precedes insulin resistance in the adipose tissue. Increased export of triglyceride would predispose to abdominal adiposity that is likely more a consequence than a cause of insulin resistance. These findings together with other data from the literature draw an intelligently attractive natural history of the metabolic syndrome and allows us to rank metabolic abnormalities linking the metabolic syndrome to cardiovascular diseases.

\section{Regulation of insulin signaling}

\section{Phosphoinositide signaling links O-GICNAc transferase to insulin resistance}

Yang X, Ongusaha PP, Miles PD, Havstad JC, Zhang F, So WV, Kudlow JE, Michell RH, Olefsky JM, Field SJ, Evans RM Howard Hughes Medical Institute and Gene Expression Laboratory, Salk Institute for Biological Studies, La Jolla, Calif., USA

Nature 2008;451:964-969

Background: Glucose flux through the hexosamine biosynthetic pathway leads to the post-translational modification of cytoplasmic and nuclear proteins by $\mathrm{O}$-linked $\beta-\mathrm{N}$-acetylglucosamine $(\mathrm{O}-\mathrm{GlcNAc})$. This tandem system serves as a nutrient sensor to couple systemic metabolic status to cellular regulation of signal transduction, transcription, and protein degradation.

Results: O-GlcNAc transferase (OGT) harbors a previously unrecognized type of phosphoinositide-binding domain. After induction with insulin, phosphatidylinositol 3,4,5-trisphosphate recruits OGT from the nucleus to the plasma membrane, where the enzyme catalyses dynamic modification of the insulinsignaling pathway by O-GlcNAc. This results in the alteration in phosphorylation of key signaling molecules and the attenuation of insulin signal transduction. Hepatic overexpression of OGT impairs the expression of insulin-responsive genes and causes insulin resistance and dyslipidemia.

Conclusion(s): These findings identify a molecular mechanism by which nutritional cues regulate insulin signaling through O-GlcNAc, and underscore the contribution of this modification to the etiology of insulin resistance and type 2 diabetes.

There are several feedback mechanisms able to attenuate insulin signaling in order to maintain glucose homeostasis. This paper tackles the mechanisms by which excess of nutrients are able to promote insulin resistance.

O-GIcNAc transferase (OGT) catalyses the attachment of O-linked $\beta$-N-acetylglucosamine (O-GIcNAc) from the glucosamine pathway to proteins, whereas O-GICNcase catalyses sugar removal, both reactions being responsible for post-translational modifications of nuclear and cytoplasmic proteins. OGT recruitment to the membrane induces phosphorylation of some components of insulin signaling, which in turn changes their activity (fig. 2). This regulatory mechanism, based on phosphorylation, results in a fine balance of activation/repression of the insulin-signaling pathway. It appears to be more active in the liver than in the muscle.

Under normal conditions, phosphatidylinositol 3,4,5-trisphosphate ( $\left.\mathrm{PIP}_{3}\right)$ recruits O-GICNAc transferase to the membrane, which in turn decreases insulin signaling by decreasing phosphorylation of Akt, and consequently Akt activity, and by increasing phosphorylation of IRS1 on Ser residues. O-GICNAC 


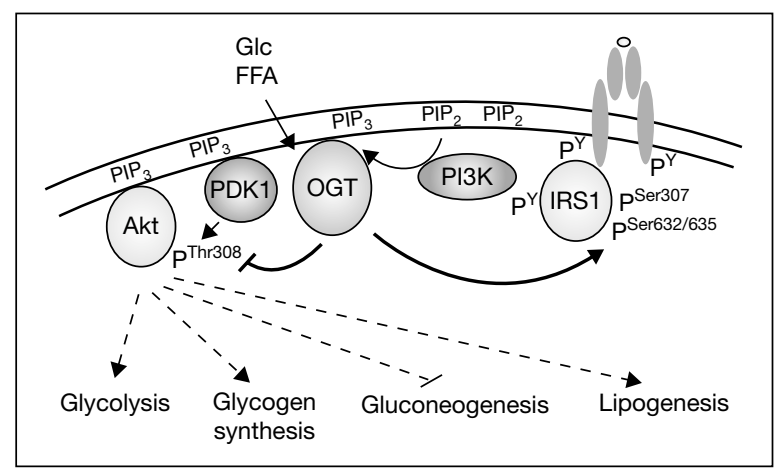

Fig. 2. Model of insulin signaling attenuation by O-GIcNAc transferase (OGT) from Yang et al. $\mathrm{PIP}_{3}=$ Phosphatidylinositol 3,4,5-trisphosphate.

transferase thus modulates the termination of insulin signaling. After activation those signals are able to promote termination of signal transduction and are of paramount importance in the insulinsignaling pathway. When there is an excess of glucose or free fatty acid (as in obesity for instance), levels of O-GIcNAc increase inducing impaired insulin action and insulin resistance. On the other hand, abnormal production of O-GIcNAc can contribute to insulin resistance.

\section{New adipo-sites}

\section{Beneficial effects of subcutaneous fat transplantation on metabolism}

Tran TT, Yamamoto Y, Gesta S, Kahn CR

Joslin Diabetes Center and Harvard Medical School, Boston, Mass., USA

Cell Metab 2008;7:410-420

Background: Subcutaneous and visceral obesity are associated with different risks of diabetes and the metabolic syndrome.

Methods: To elucidate whether these differences are due to anatomic location or intrinsic differences in adipose depots, mice were studied 12 weeks after transplantation of subcutaneous fat or visceral fat from donor mice into either subcutaneous or visceral regions of recipient mice.

Results: The group with subcutaneous fat transplanted into the visceral cavity exhibited decreased body weight, total fat mass, and glucose and insulin levels. Insulin sensitivity was also improved with increased whole-body glucose uptake, glucose uptake into endogenous fat, and insulin suppression of hepatic glucose production. These effects were observed to a lesser extent with subcutaneous fat transplanted to the subcutaneous area, whereas visceral fat transplanted to the visceral area was without effect.

Conclusion(s): These data suggest that subcutaneous fat is intrinsically different from visceral fat and produces substances that can act systemically to improve glucose metabolism.

Although published in 2008, should Claude Bernard have reviewed this paper, he would certainly have accepted it! It is a beautiful example of physiology with nothing fancy about the technology. The field of the paper is one of the most debated in the field of obesity and insulin resistance: who is the culprit, subcutaneous or visceral fat? The data clearly show that subcutaneous fat is not harmful and even more it is responsible for a more favorable metabolic profile. A letter published in Diabetologia this year confirms this observation [15]. The reason for this effect is not clear but the results show that subcutaneous fat can cross-talk with first the other fat depots and second with other organs involved in the process of insulin resistance such as the liver. The messengers have not 
been identified and they are not leptin or adiponectin. The cross-talk of the adipose tissue with other organs was first described with the adipocyte-specific GLUT4 KO, model in which invalidation of glucose transport in the adipocytes induced peripheral and muscle insulin resistance [16]. These data extend the first clinical observation dated back to 1947 by Jean Vague who reported that upper body fat ('apple' body shape) was not deleterious from the metabolic perspective in contrast to central accumulation ('pear' body shape) [17].

\title{
New gene
}

\section{Mechanisms by which common variants in the TCF7L2 gene increase risk of type 2 diabetes}

\author{
Lyssenko V, Lupi R, Marchetti P, Del Guerra S, Orho-Melander M, Almgren P, Sjogren M, Ling C, Eriksson KF, \\ Lethagen AL, Mancarella R, Berglund G, Tuomi T, Nilsson P, Del Prato S, Groop L \\ Department of Clinical Sciences, Diabetes and Endocrinology, and Lund University Diabetes Center, Lund University, \\ Malmo, Sweden
}

J Clin Invest 2007;117:2155-2163

Background: Genetic variants in the gene encoding for transcription factor-7-like 2 (TCF7L2) have been associated with type 2 diabetes (T2D) and impaired $\beta$-cell function, but the mechanisms have remained unknown.

Methods: This is a prospective study on the ability of common variants in TCF7L2 to predict future T2D and explored the mechanisms by which they would do this. Scandinavian subjects followed for up to 22 years were genotyped for 3 SNPs (rs7903146, rs12255372, and rs10885406) in TCF7L2, and a subset of them underwent extensive metabolic studies. Expression of TCF7L2 was related to genotype and metabolic parameters in human islets.

Results: The CT/TT genotypes of SNP rs7903146 strongly predicted future T2D in 2 independent cohorts (Swedish and Finnish). The risk T allele was associated with impaired insulin secretion, incretin effects, and anenhanced rate of hepatic glucose production. TCF7L2 expression in human islets was increased in T2D, particularly in carriers of the TT genotype. Overexpression of TCF7L2 in human islets reduced glucose-stimulated insulin secretion.

Conclusion(s): The increased risk of T2D conferred by variants in TCF7L2 involves the enteroinsular axis, enhanced expression of the gene in islets, and impaired insulin secretion.

TCF7L2 encodes for a transcription factor in the Wnt signaling pathway, inducing transcription of a number of genes when hetrodimerized, among which are proglucagon and GLP-1 [18]. TCF7L2 is one of the few SNPs strongly associated with T2D in all genome-wide association studies. Several other studies have suggested that the increased risk of diabetes is mediated by impaired insulin secretion. An incretin effect (through the secretion or the effect of GLP-1) has been suspected.

This paper shows the following:

- The association of the mutated allele with the risk of developing diabetes has been based, for the first time, on a prospective follow-up. Data established through a longitudinal observation are more robust than the ones based on a cross-sectional association study.

- This increased risk is mediated by an impaired insulin secretion and not by an effect on BMI or insulin resistance.

- The reason for the impaired insulin secretion is not univocal. Some of the clinical data point to an incretin effect. Direct evidence at the level of the pancreatic islets suggests a primary effect, not mediated through the diabetic state, on the insulin gene expression or on $\beta$-cell proliferation. Surprisingly, the expressed TCF $\angle 2$ is increased 5 -fold in the diabetic pancreas in comparison to controls and overexpression of TCF7L2 in human islets leads to a reduction in insulin secretion. It is likely that the mutated risk allele results in overexpression of TCF7L2, which in turn reduces insulin secretion. 


\section{The human microbiome project}

Turnbaugh PJ, Ley RE, Hamady M, Fraser-Liggett CM, Knight R, Gordon J

Center for Genome Sciences, Washington University School of Medicine, St Louis, Mo., USA

Nature 2007;449:804-810

The microorganisms that live inside and on the humans (known as microbiota) appear more and more important for human health and diseases (sources of energy and vitamins, metabolic phenotypes, immune system, behavior, etc.). The Human Genome Project, supported by the NIH, aims at understanding the microbial components of the human genetic and metabolic landscape and how they contribute to normal physiology and predisposition to disease. To achieve this goal, there is a need for sequencing reference microbial genomes and linking short gene fragments to organisms. On the human side, 'normal' states need to be defined before the effect of the microbiota can be evaluated.

Recent data showing that obesity can be correlated with the degree of representation of microbial genes involved in certain metabolic pathways has focused on the need to study further the genome of our microbial symbionts (the microorganisms that live inside and on humans and thus are called the microbiome). To understand the range of human genetic and physiological diversity, the microbiome and the factors that influence the distribution of the consistent microorganisms must be characterized. The suspected functional contributions of the gut microbiota are numerous and different. It could change the energy value of food by modulating the digestive capacity of an individual. This could have implications for identifying individuals who are at risk of malnourishment or obesity. The microbiota could also be a (largely unexplored) regulator of drug metabolism and bioavailability. The detoxifying function of ingested carcinogens, for example, may affect a host's susceptibility. Gut microbiota could be involved in the renewal of gut epithelial cells, the development of the immune system or even in changes in behavior!! Studying the microbiome is complex as its biogeography is as different on the individual level as it is on the population level. Moreover, the proportion of the various subgroups of genes is different between gut and environmental bacteria. It is highly complex to define the microbiome of a 'healthy' subject, but this is the first step of this project. The aim of this international network is both very ambitious and exciting. Many outcomes can be anticipated such as new diagnostic biomarkers of health or a pharmacopoeia that includes members of the human microbiota and the chemical messengers they produce. One important outcome is anticipated to be a better understanding of the nutritional requirements of humans. This could result in new recommendations for food production and consumption that are formulated based on knowledge of our microbiome.

\section{Food for thought}

\section{Metabolic endotoxemia initiates obesity and insulin resistance}

Cani PD, Amar J, Iglesias MA, Poggi M, Knauf C, Bastelica D, Neyrinck AM, Fava F, Tuohy KM, Chabo C, Waget A, Delmee E, Cousin B, Sulpice T, Chamontin B, Ferrieres J, Tanti JF, Gibson GR, Casteilla L, Delzenne NM, Alessi MC, Burcelin $\mathrm{R}$ Institute of Molecular Medicine, I2MR Toulouse, France

Diabetes 2007;56:1761-1772

Background: Diabetes and obesity are two metabolic diseases characterized by insulin resistance and a lowgrade inflammation. Seeking an inflammatory factor causative of the onset of insulin resistance, obesity, and diabetes, the authors have identified bacterial lipopolysaccharide (LPS) as a triggering factor.

Results: The authors found that normal endotoxemia increased or decreased during the fed or fasted state, respectively, on a nutritional basis and that a 4-week high-fat diet chronically increased the plasma LPS concentration two to three times, a threshold that we have defined as metabolic endotoxemia. Importantly, a high-fat diet increased the proportion of a LPS-containing microbiota in the gut. When metabolic endotoxemia was induced for 4 weeks in mice through continuous subcutaneous infusion 
of LPS, fasted glycemia and insulinemia and whole-body, liver, and adipose tissue weight gain were increased to a similar extent as in mice fed a high-fat diet. In addition, adipose tissue F4/80-positive cells, markers of inflammation, and liver triglyceride content were increased. Furthermore, liver, but not whole-body, insulin resistance was detected in LPS-infused mice. CD14 mutant mice resisted most of the LPS and high-fat diet-induced features of metabolic diseases. This new finding demonstrates that metabolic endotoxemia dysregulates the inflammatory tone and triggers body weight gain and diabetes. Conclusion(s): The LPS/CD14 system sets the tone of insulin sensitivity and the onset of diabetes and obesity. Lowering the plasma LPS concentration could be a potent strategy for the control of metabolic diseases.

This article illustrates the interaction between gut microbiota, diet and metabolic diseases eluded to in the previous review. In their quest to determine triggering factors of the early development of obesity and insulin resistance the authors identified bacterial lipopolysaccharide (LPS) as a good candidate. LPS is a strong stimulator of the release of several cytokines that are involved in the development of insulin resistance associated with obesity. It is continuously produced in the gut by the death of gram-negative bacteria and physiologically translocated into intestinal capillaries. The authors demonstrate that, under nutritional conditions leading to obesity, the changes in gut microbiota which induce increased LPS translocation not only participate but also increase inflammation, weight gain and liver insulin resistance associated with a high-fat diet. This action seems to be mediated by macrophages which would be activated by LPS. This is the first direct report of a gut microbiotic product that stimulates weight gain and insulin resistance. Taken together with data suggesting that antibiotic treatment could protect against autoimmune diabetes by decreasing inflammation, this article opens a new field of therapeutic intervention to prevent insulin resistance or weight gain.

References

1. Schwartz MW, Woods SC, Porte D Jr, Seeley RJ, Baskin DG: Central nervous system control of food intake. Nature 2000;404:661-671.

2. Bady I, Marty N, Dallaporta M, Emery M, Gyger J, Tarussio D, et al: Evidence from glut2-null mice that glucose is a critical physiological regulator of feeding. Diabetes 2006;55:988-995.

3. Kang L, Dunn-Meynell AA, Routh VH, Gaspers LD, Nagata Y, Nishimura T, et al: Glucokinase is a critical regulator of ventromedial hypothalamic neuronal glucosensing. Diabetes 2006;55:412-420.

4. Wang R, Liu X, Hentges ST, Dunn-Meynell AA, Levin BE, Wang W, et al: The regulation of glucose-excited neurons in the hypothalamic arcuate nucleus by glucose and feeding-relevant peptides. Diabetes 2004;53:1959-1965.

5. Abdul-Ghani MA, Jenkinson CP, Richardson DK, Tripathy D, DeFronzo RA: Insulin secretion and action in subjects with impaired fasting glucose and impaired glucose tolerance: results from the Veterans Administration Genetic Epidemiology Study. Diabetes 2006;55:1430-1435.

6. Faerch K, Vaag A, Holst JJ, Glumer C, Pedersen O, Borch-Johnsen K: Impaired fasting glycaemia vs impaired glucose tolerance: similar impairment of pancreatic alpha and beta cell function but differential roles of incretin hormones and insulin action. Diabetologia 2008;51:853-861.

7. Meyer C, Pimenta W, Woerle HJ, Van Haeften T, Szoke E, Mitrakou A, et al: Different mechanisms for impaired fasting glucose and impaired postprandial glucose tolerance in humans. Diabetes Care 2006;29:1909-1914.

8. Druet C, Levy-Marchal C: Insulin resistance, the metabolic syndrome and type 2 diabetes; in Carel J, Hochberg Z (eds): Yearbook of Pediatric Endocrinology. Basel, Karger, 2007, pp 133-145.

9. Levy-Marchal C: Insulin resistance, the metabolic syndrome and type 2 diabetes; in Carel J, Hochberg Z (eds): Yearbook of Pediatric Endocrinology. Basel, Karger, 2006, pp 149-162.

10. Huang TT, Nansel TR, Belsheim AR, Morrison JA: Sensitivity, specificity, and predictive values of pediatric metabolic syndrome components in relation to adult metabolic syndrome: the Princeton LRC follow-up study. J Pediatr 2008;152:185-190.

11. Pankow JS, Jacobs DR Jr, Steinberger J, Moran A, Sinaiko AR: Insulin resistance and cardiovascular disease risk factors in children of parents with the insulin resistance (metabolic) syndrome. Diabetes Care 2004;27:775-780.

12. Dabelea D, Hanson RL, Lindsay RS, Pettitt DJ, Imperatore G, Gabir MM, et al: Intrauterine exposure to diabetes conveys risks for type 2 diabetes and obesity: a study of discordant sibships. Diabetes 2000;49:2208-2211.

13. Sobngwi E, Boudou P, Mauvais-Jarvis F, Leblanc H, Velho G, Vexiau P, et al: Effect of a diabetic environment in utero on predisposition to type 2 diabetes. Lancet 2003;361:1861-1865.

14. Guzzaloni G, Grugni G, Minocci A, Moro D, Morabito F: Liver steatosis in juvenile obesity: correlations with lipid profile, hepatic biochemical parameters and glycemic and insulinemic responses to an oral glucose tolerance test. Int J Obes Relat Metab Disord 2000;24:772-776.

15. Hocking SL, Chisholm DJ, James DE: Studies of regional adipose transplantation reveal a unique and beneficial interaction between subcutaneous adipose tissue and the intra-abdominal compartment. Diabetologia 2008;51:900-902.

16. Abel ED, Peroni O, Kim JK, Kim YB, Boss O, Hadro E, et al: Adipose-selective targeting of the GLUT4 gene impairs insulin action in muscle and liver. Nature 2001;409:729-733.

17. Vague J: La différenciation sexuelle, facteur déterminant des formes de l'obésité. Presse Méd 1947;30:339-340.

18. Ong KK: Population genetics and pharmacogenetics; in Carel J, Hochberg Z (eds): Yearbook of Pediatric Endocrinology. Basel, Karger, 2007, pp 147-159. 